\title{
Indications and considerations for kidney biopsy: an overview of clinical considerations for the non-specialist
}

\author{
Authors: Katherine L Hull, ${ }^{A *}$ Sherna F Adenwalla, ${ }^{B *}$ Peter Topham ${ }^{C}$ and Matthew P Graham-Brown ${ }^{D}$
}

\begin{abstract}
Around 3 million people in the UK have chronic kidney disease and $20 \%$ of hospital admissions are complicated by acute kidney injury. Decline in kidney function is not a diagnosis; it is essential to identify and treat underlying causes of acute and chronic kidney disease to either achieve recovery or slow the decline of kidney function. Thorough clinical assessment and simple investigations help determine the category of kidney injury (pre-renal, intrinsic or post-renal) and inform the need for kidney biopsy, which can provide significant information in the evaluation of suspected intrinsic kidney disease, supporting diagnosis, guiding prognosis and management, and identifying disease relapse. The procedure is invasive and not without risk, which although small has the potential to be both organ- and life-threatening. This review outlines roles of kidney biopsy for the non-specialist, with focus of its role in patients with diabetes, lupus, myeloma and in the older patient.
\end{abstract}

KEYWORDS: kidney biopsy, chronic kidney disease, acute kidney injury, haematuria, proteinuria

DOI: $10.7861 /$ clinmed.2021-0472

\section{Introduction}

Despite advances in non-invasive biochemical and imaging investigations, kidney biopsies play a pivotal role in the diagnosis of kidney disease. They provide prognostic information that may result in treatment modification in up to $74 \%$ of patients. ${ }^{1}$ This article summarises common indications for kidney biopsy in acute and chronic presentations of kidney disease for non-specialists. This review is limited to the use of percutaneous kidney biopsies

Authors: ${ }^{\text {A }}$ linical research fellow and specialist registrar in nephrology, University Hospitals of Leicester NHS Trust, Leicester, UK and University of Leicester, Leicester, UK; ${ }^{B}$ academic clinical fellow and internal medical trainee, University Hospitals of Leicester NHS Trust, Leicester, UK and University of Leicester, Leicester, UK; ${ }^{C}$ consultant nephrologist, University Hospitals of Leicester NHS Trust, Leicester, UK; ${ }^{D}$ NIHR academic clinical lecturer in renal medicine and consultant nephrologist, University Hospitals of Leicester NHS Trust, Leicester, UK and University of Leicester, Leicester, UK; * joint first authors for the diagnosis and management of native parenchymal kidney disease in adults, not for the diagnosis of tumours or growths, biopsies in children, or kidney transplant recipients.

\section{Identifying people who may need a kidney biopsy}

Urinary 'sediment'

Urinalysis with a urine dipstick is an essential initial investigation for acute and chronic presentations of kidney disease, as well as for monitoring patients with known kidney disease. The presence of blood and/or protein suggests inflammation in the kidney and damage to the filtration barrier, indicating the origin of the inflammation may be the glomerulus. ${ }^{2}$ Minor urine dipstick abnormalities (such as low-grade proteinuria, absent or minimal haematuria, with or without leucocytes) in the presence of deranged kidney function may suggest tubulointerstitial nephritis. In addition to urinalysis, a decline in kidney function should prompt evaluation with investigations listed in Table 1. The

Table 1. Summary of the essential investigations to evaluate a decline in kidney function

$\begin{array}{ll}\text { Blood tests } & \text { Renal profile with eGFR } \\ & \text { Full blood count } \\ & \text { Bone profile } \\ \text { Bedside } & \text { Blood pressure } \\ \text { Urine } & \text { Urine dipstick for blood, protein, nitrites and } \\ & \text { leucocytes } \\ & \text { Urine culture and sensitivity } \\ & \text { Urine protein-creatinine ratio } \\ & \text { Urine albumin-creatinine ratio } \\ \text { Imaging } & \begin{array}{l}\text { Ultrasound: kidney, ureters and bladder } \\ \text { (to assess kidney size; for masses, cysts or }\end{array} \\ \text { hydronephrosis) } & \text { Immunoglobulins and electrophoresis, ANCA, } \\ \text { C3 and C4, ANA, dsDNA antibody, anti-GBM } \\ \text { antibody, anti-PLA2R antibody }\end{array}$




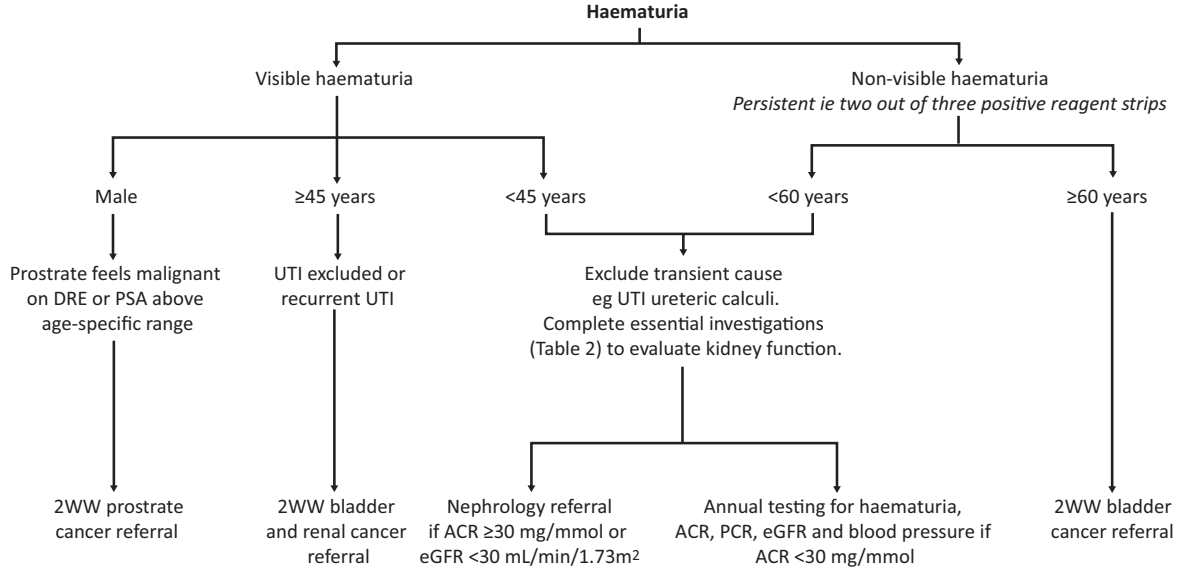

Fig 1. Haematuria assessment pathway, adapted from NICE urological cancers clinical knowledge summary and NICE chronic kidney disease guidelines. ${ }^{3,4} \mathrm{ACR}$ = albumin-creatinine ratio; DRE $=$ digital rectal examination; eGFR $=$ estimated glomerular filtration rate; NICE = National Institute for Health and Care Excellence; $P C R=$ protein-creatinine ratio; $P S A=$ prostate specific antigen; $2 \mathrm{WW}=2$-week wait; UTI = urinary tract infection. immunological screen is particularly important in patients with acute kidney injury (AKI) and an active urinary sediment (ie blood and protein on urine dipstick).

\section{Haematuria}

Haematuria may be classified as 'visible' or 'non-visible'. Both can be due to nephrological (eg glomerulonephritis) or urological (eg malignancy, infection or calculi) pathology, and clinical presentation will guide referral. Fig 1 summarises an evaluation process for both visible and non-visible haematuria, adapted from the National Institute for Health and Care Excellence (NICE) guidelines. ${ }^{3,4}$

\section{Malignancy}

Urological malignancy can cause both visible and non-visible haematuria. History is important in identifying risk factors: smoking status, drug history (eg cyclophosphamide), occupation, chemical exposure and travel history (eg schistosomiasis)., Urgent referral for the assessment of urological malignancy is guided by age (Fig 1). Once malignancy has been excluded, other causes should be considered.

\section{Visible haematuria}

A single episode of visible haematuria warrants investigation. ${ }^{7}$ Transient causes should be excluded by rechecking a urine dipstick after an acute episode has resolved. Anticoagulant and antiplatelet therapy will worsen any haematuria but will not be the precipitant.

The appearance of visible haematuria is a useful indicator to the origin of pathology. Pink stained urine, or frank blood, suggests fresh bleeding along the urinary tract and points towards urological causes. In contrast, visible haematuria that is dark ('cola-coloured') is suggestive of a nephrological cause due to haemoglobin being converted to methaemoglobin in the acidic environment.

Co-existent symptoms also provide information. In urological pathology, flank pain suggests ureteric colic, while intercurrent illness (typically upper respiratory tract infection) followed by cola-coloured urine is suggestive of a nephrological cause (eg post-infectious glomerulonephritis or immunoglobulin (Ig) A nephropathy).
Isolated non-visible haematuria with normal renal function Kidney biopsy to investigate isolated non-visible haematuria, in the absence of proteinuria and renal impairment, with normotension, is unlikely to change management. ${ }^{8,9}$ Thinbasement membrane nephropathy and IgA nephropathy are frequent causes. ${ }^{8,10}$ However, persistent isolated non-visible haematuria has been associated with a significant, albeit small, incidence of end-stage kidney disease (ESKD). ${ }^{11,12}$ Annual monitoring in primary care with blood pressure, urinalysis and serum creatinine levels is necessary.

Biopsy can assist in the diagnosis of inherited conditions and prompt screening of relatives, genetics referral and monitoring. Thin-basement membrane nephropathy usually has a good prognosis with only a minority progressing to ESKD, and so clear diagnosis can provide reassurance and avoid unnecessary further investigation. ${ }^{12}$ Alport's syndrome has a lower prevalence but greater risk of progression to ESKD, especially in males, and has extra-renal manifestations, such as hearing impairment. ${ }^{12}$ Early diagnosis and family screening to identify affected family is essential.

\section{Non-visible haematuria with markers of abnormal renal function}

Non-visible haematuria with markers of abnormal kidney function (such as increasing serum creatinine, reduced urine output, hypertension or proteinuria) has a variety of potential urological and nephrological causes.

Regarding intrinsic kidney disease, non-visible haematuria with deranged kidney function often reflects glomerular inflammation (glomerulonephritis). These patients may be systemically unwell and, in the presence of an acute decline in their kidney function, will require close monitoring and follow-up. Kidney biopsy is a key component to diagnosis, management and prognosis, whereby the focus of treatment is the underlying glomerulonephritis, supportive care to manage to complications (eg fluid overload) and to preserve kidney function (eg blood pressure management). ${ }^{13}$ Biopsy may be postponed or not completed if unlikely to change management, if the patient is high risk or to avoid treatment delays if the diagnosis is clear. For example, in anti-glomerular basement membrane disease, the presence of circulating 
antibodies, rapidly progressive AKI and haematuria (with or without pulmonary haemorrhage) confirms the diagnosis. ${ }^{14}$

\section{Proteinuria}

Proteinuria should be quantified using both the urinary protein creatinine ratio (PCR) and albumin-creatinine ratio (ACR). The presence of albuminuria, as defined by an $A C R \geq 3 \mathrm{mg} / \mathrm{mmol}$, is preferred in the detection and management of chronic kidney disease (CKD) due to its prognostic value and reflection of glomerular injury. Even with an estimated glomerular filtration rate (eGFR) within expected limits, the presence of albuminuria for $>3$ months reflects persistent glomerular dysfunction and CKD. ${ }^{15}$ Albuminuria can be subdivided into microalbuminuria (a moderate increase in ACR of $\geq 3$ to $\leq 30 \mathrm{mg} / \mathrm{mmol}$ ) and macroalbuminuria (a severe increase in ACR of $>30 \mathrm{mg} / \mathrm{mmol}$ ) influencing decisions regarding monitoring and referral. ${ }^{15}$ Urinary ACR is an essential investigation when assessing new kidney disease, known CKD and those at risk of kidney damage. This is because urinary ACR will detect early, low-grade proteinuria that has a high risk of being missed on urine dipstick. ${ }^{4}$

It is important to note that proteinuria can occur as the result of excess serum free light chains or impaired absorption in the proximal tubule. This results in proteinuria with minimal albuminuria (see the myeloma section later), highlighting the risk of false negative results if urine ACR is used in isolation. ${ }^{16}$

Unless there is a clear history of kidney disease secondary to systemic illness (eg diabetes mellitus and hypertension) or the risks of biopsy outweigh the benefits, biopsy in patients with unexplained proteinuria provides valuable information. The presence of proteinuria should be confirmed on an early morning urine sample. Benign phenomena (such as orthostatic (ie postural) proteinuria) typically occur in individuals $<30$ years old and presents as isolated proteinuria; the absence of proteinuria on an early morning sample confirms the diagnosis and further investigation is not indicated. 17,18

Nephrotic range proteinuria is defined as a urinary PCR $>300 \mathrm{mg} / \mathrm{mmol}$ or ACR $>220 \mathrm{mg} / \mathrm{mmol}$, and may or may not occur with the nephrotic syndrome: heavy proteinuria in combination with hypoalbuminaemia $(<25 \mathrm{~g} / \mathrm{L})$ and oedema, with or without significant hypercholesterolaemia $(>10 \mathrm{mmol} / \mathrm{L}){ }^{15}$ Nephrotic syndrome is a clinical presentation and the underlying diagnosis should be sought. There are some situations associated with nephrotic syndrome (such as in diabetic kidney disease or amyloidosis) where clinical features and less invasive investigations can indicate the diagnosis and biopsy can be avoided. However, if common clinical diagnoses have been ruled out, it would be important to consider biopsy in adults.

\section{Kidney biopsy in chronic kidney disease vs acute kidney injury}

Kidney biopsy is a useful diagnostic tool in both the acute and chronic setting, especially in the presence of active urinary sediment. However, active urinary sediment does not confirm intrinsic kidney disease and biopsy poses unnecessary risk in cases of pre-renal and post-renal causes of kidney impairment. Furthermore, an active urine sediment is not a prerequisite for additional investigation; kidney biopsy may also be appropriate in cases of bland urinary sediment, for instance, non-recovering AKI and suspected tubulointerstitial nephritis.

\section{Specific situations when kidney biopsies may be considered}

\section{Diabetic kidney disease}

Thirty per cent to $40 \%$ of people with type 1 and type 2 diabetes mellitus (DM) in the UK develop CKD and their risk of requiring renal replacement therapy is over three times the general population. 19,20 'Diabetic kidney disease' refers to the structural and functional changes caused by DM, while 'diabetic nephropathy' refers to specific histological findings on biopsy. Alternative or superadded diagnoses may co-exist (eg hypertensive disease, unresolved AKI or glomerulonephritis). ${ }^{21}$ The distinction is important for treatment, prognosis and future transplant decisions.

The natural history of diabetic kidney disease in type 1 and type $2 \mathrm{DM}$ is well defined and the development of albuminuria is a consistent predictor for progression to ESKD. ${ }^{22,23}$ In most patients, clinical history, course of disease and non-invasive investigations can identify where CKD is likely a consequence of DM. National Kidney Foundation guidelines advise that CKD can be attributed to DM in the presence of macroalbuminuria or microalbuminuria with retinopathy, or the presence of microalbuminuria in patients with type $1 \mathrm{DM}$ for $>10$ years. ${ }^{24}$ In these instances, the risks of biopsy are thought to outweigh the benefits of a confirmatory diagnosis. Features suggestive of non-diabetic causes of kidney damage that should prompt further investigation (Table 1) include:

$>$ refractory hypertension or large drop in renal function following initiation of renin-angiotensin-aldosterone system inhibitors (suggestive of reno-vascular disease)

$>$ the absence of diabetic retinopathy in the presence of proteinuria is predictive for non-diabetic kidney disease and strengthens the need for further investigation

$>$ haematuria

$>$ evidence of other systemic disease. ${ }^{24,25}$

Additionally, rapidly decreasing kidney function and rapidly increasing proteinuria / nephrotic-range proteinuria may occur due to DM or could indicate an additional diagnoses; further investigation (Table 1) and referral to nephrology would be warranted.

\section{Lupus nephritis}

Lupus nephritis (LN) occurs in approximately $50 \%$ of patients with systemic lupus erythematosus (SLE). ${ }^{26}$ Patients may lack overt clinical signs of kidney disease and monitoring kidney function and urinary sediment are important. ${ }^{27}$ The gold standard for diagnosis of LN is kidney biopsy and earlier biopsy is associated with improved outcomes. ${ }^{28}$ The role for repeat biopsy in the disease course is also relatively clear.

\section{Initial presentation}

LN should be considered in any patient with SLE with declining renal function, proteinuria $>0.5 \mathrm{~g}$ per 24 hours or active urinary sediment. ${ }^{29,30}$ Each histological class is associated with different treatment decisions and prognosis; thus, timely biopsy and 
re-biopsy are essential investigations for both diagnosis and to guide management. ${ }^{31}$ Biopsy also serves to detect alternative causes of kidney damage in SLE (such as druginduced nephrotoxicity, lupus podocytopathy or thrombotic microangiopathy).

\section{Repeat biopsy}

Biopsy can be used to diagnose relapses or progression of disease. Histological transformation can occur with relapses, potentially changing the treatment required and prognosis. ${ }^{30,31}$ Furthermore, relapses are an independent predictor of progression to ESKD. ${ }^{32}$ In patients where relapse is suspected, there is a low threshold for repeat biopsy. ${ }^{29}$ There are no accurate clinical predictors of class transformation, reinforcing the value in histologically restaging the disease to guide immunosuppression and inform the risk of progression to ESKD. ${ }^{33,34}$

\section{The older patient}

The average age of someone hospitalised with AKI is 76 years. ${ }^{35}$ Approximately $54 \%$ of people aged $>75$ years live with CKD 3-5, with clear comorbid consequences. ${ }^{36}$

GFR declines physiologically with age. ${ }^{37}$ Identifying patients with pathological, non-senescent kidney disease remains challenging. The presence of abnormal urinary sediment warrants investigation, as outlined earlier.

Histological findings may highlight a discrepancy in the clinical diagnosis in up to a third of older patients. ${ }^{38}$ In a recent UK retrospective cohort study of biopsies in patients aged $>70$ years, scarring due to diabetes and hypertension was identified in $36 \%$ while the remainder included pauci-immune glomerulonephritis (12\%), tubulointerstitial nephritis (11\%), membranous glomerulonephritis (7\%) and other diagnoses. ${ }^{39}$ Appropriate treatment with immunosuppression in this group can still alter progression to ESKD and survival. ${ }^{40}$ Biopsies also provide prognostic information; those diagnosed with vasculitis and paraprotein-related renal disease had the highest risk of progression to ESKD compared with other diagnoses. ${ }^{39}$ Prognostic information facilitates earlier discussions around conservative care or initiation of dialysis. Some studies have found that older patients have an increased risk of bleeding post-biopsy, while others have observed no increased risk compared with other age groups. ${ }^{41,42}$

As with all data from registries, case series or retrospective cohorts, there will be an element of selection bias involving the population under investigation. However, the findings from these studies highlight that if these patients fit the criteria for requiring a kidney biopsy, then it is generally safe to proceed and age itself should not be an exclusion criterion.

\section{Myeloma}

Multiple myeloma is a malignancy of bone marrow plasma cells and is characterised by the clonal proliferation of plasma cells (derived from B cells) and subsequent production of a monoclonal paraprotein. ${ }^{43}$ Diagnosis involves the presence of end-organ damage attributable to the plasma cell proliferation: hypercalcaemia, bone lesions, anaemia or renal insufficiency. 44,45 Up to a third of individuals have kidney impairment at the time of myeloma diagnosis. ${ }^{46}$ Kidney injury can occur directly (eg light-chain cast nephropathy, immunoglobulin deposition disease or amyloidosis) or indirectly due to sepsis, dehydration, hypercalcaemia or medication toxicity. ${ }^{44,47,48}$ There may be preexisting CKD secondary to conditions such as hypertension or DM. ${ }^{45,48}$ Light-chain cast nephropathy is considered a 'myelomadefining' event. ${ }^{45}$ Urinalysis will demonstrate proteinuria due to the presence of filtered light chains (Bence-Jones protein), with minimal albuminuria as the glomerular basement membrane is intact. ${ }^{48,49}$ Histological diagnosis confirms the presence of lightchain cast nephropathy, however, a presumptive diagnosis can be made with high serum free light chain levels $(>1,500 \mathrm{mg} / \mathrm{L})$ and AKI; a kidney biopsy should not delay treatment in such cases. ${ }^{45}$ Biopsy should be considered when a diagnosis is uncertain (for instance, when serum free light chain levels are $<500 \mathrm{mg} / \mathrm{L}$ ) to exclude other causes of AKI and CKD. ${ }^{45,47}$

Plasma cell dyscrasias in the absence of end-organ damage are termed monoclonal gammopathy of undetermined significance (MGUS) and considered pre-malignant conditions. ${ }^{45}$ MGUS can lead to kidney injury through glomerular and tubule dysfunction. The term 'monoclonal gammopathy of renal significance' (MGRS) has been coined to discriminate non-myeloma plasma cell dyscrasias that result in kidney injury. ${ }^{50}$ The prognostic significance of biopsy findings in plasma cell dyscrasias is unclear. ${ }^{51}$ Ultimately, management should be focused on the underlying plasma cell dyscrasia; achieving haematological response is associated with both overall and kidneyspecific survival in multiple myeloma and $\mathrm{AL}$ amyloidosis. ${ }^{49,52,53}$

\section{Safety of the procedure}

Kidney biopsies are invasive procedures and not without risk. The decision to proceed requires oversight (and usually completion) from the nephrology team and a shared decision with the patient. Biopsies should only be performed when the results will guide treatment, assist with diagnosis that will alter treatment or inform prognosis. Meticulous preparation of the patient is key and important contraindications to biopsy are outlined in Table 2. Ultrasound is important prior to biopsy to ensure that the kidneys can be visualised, to rule out anatomical abnormalities and to provide further information to determine the risk-benefit balance of the procedure. For instance, small kidneys and poor corticomedullary differentiation indicate (unquantifiable) chronicity of the renal disease and potential for limited reversibility, and there may be challenges in differentiating the kidneys from surrounding retroperitoneal structures. Patients may be concerned about the effect of removing kidney tissue on kidney function. Reassuringly, one study estimated that, in stable transplant patients, the GFR loss due to biopsy is $0.77 \mathrm{~mL} / \mathrm{min}^{54}$

Major complications from kidney biopsy are related to bleeding: haematoma formation (11\%); bleeding requiring transfusion (1.6\%); pain (4.3\%); macroscopic haematuria (3.5\%); and, rarely, death $(0.06 \%) .{ }^{55}$ Candidates with hypertension, high creatinine, thrombocytopenia, anaemia or requiring early recommencement of anticoagulation are at higher risk of severe bleeding; if these cannot be corrected pre-biopsy and biopsy is essential, then close monitoring is recommended with additional risks discussed with the patient. ${ }^{56-58}$ Patients should be monitored for $6-8$ hours post-procedure and higher risk candidates may be admitted overnight. Any haematuria post-biopsy warrants admission to gain intravenous access and assess full blood count. Computed tomography angiography may be required to identify active bleeding points amenable to endovascular intervention. 


\begin{tabular}{|c|c|}
\hline Contraindication & Reason \\
\hline \multicolumn{2}{|l|}{ Relative } \\
\hline Hypertension & Increased risk of bleeding \\
\hline Coagulopathy & $\begin{array}{l}\text { Should be corrected prior to biopsy; } \\
\text { antiplatelets and anticoagulants } \\
\text { should be held }\end{array}$ \\
\hline Renal asymmetry & $\begin{array}{l}\text { Suggests a process affecting the } \\
\text { kidneys differentially eg renal artery } \\
\text { stenosis }\end{array}$ \\
\hline $\begin{array}{l}\text { Small kidneys on } \\
\text { imaging }\end{array}$ & $\begin{array}{l}\text { Suggests damage is chronic and } \\
\text { irreversible; would be technically } \\
\text { difficult and nonspecific fibrotic } \\
\text { changes are likely to be found on } \\
\text { biopsy }\end{array}$ \\
\hline Single kidney & $\begin{array}{l}\text { Single kidneys are not routinely } \\
\text { biopsied due to the risk of } \\
\text { complications leaving the patient } \\
\text { with renal failure; however, a biopsy } \\
\text { may be justified if there is a chance } \\
\text { of discovering a treatable cause of } \\
\text { kidney injury that would otherwise } \\
\text { cause end-stage disease }\end{array}$ \\
\hline \multicolumn{2}{|l|}{ Absolute } \\
\hline $\begin{array}{l}\text { Active pyelonephritis or } \\
\text { skin infection at site of } \\
\text { needle insertion }\end{array}$ & Increased risk of sepsis \\
\hline $\begin{array}{l}\text { Uncontrollable } \\
\text { hypertension or } \\
\text { coagulopathy }\end{array}$ & Increased risk of bleeding \\
\hline $\begin{array}{l}\text { Unable to tolerate the } \\
\text { procedure }\end{array}$ & \\
\hline
\end{tabular}

It is important to note that histological samples do not always provide a definitive answer and the findings should be interpreted in the context of the history and other investigations. Accurate diagnosis and prognostication can be affected by sampling error, especially in focal pathologies, or where too few glomeruli have been captured. Histological findings are not always specific; for example, interstitial fibrosis and tubular atrophy are signs of chronic damage, rather than pointing towards a diagnosis.

\section{Conclusion}

Kidney biopsy is key tool in the evaluation of both AKI, CKD and established intrinsic kidney disease to guide management strategies and confirm diagnoses. It is a procedure not without risk, and so it is essential that preliminary investigations are completed to guide differential diagnoses and secondary care referral, with the exclusion of pre-renal and post-renal causes prior to biopsy completion. Although important, the kidney biopsy should not delay treatment when the diagnosis is established from other investigations.

\section{Key points}

> Kidney biopsy can be a valuable investigation for acute kidney injury and chronic kidney disease for the diagnosis of intrinsic renal disease and to guide prognosis and management.

> Kidney biopsy is an invasive procedure that carries serious, albeit low probability, risks and there are a number of contraindications to consider and should always be undertaken with specialist nephrology input.

> Simple investigations, such as serum creatinine, blood pressure, ultrasound of the urinary tract, urine samples (dipstick, PCR, ACR and culture) and a renal immunological screen will assist in guiding whether kidney biopsy is indicated.

$>$ Often it is not appropriate to delay treatment to allow for a kidney biopsy if the underlying diagnosis is clear.

> A patient-centred approach should be adopted when considering whether a kidney biopsy is appropriate (eg isolated non-visible haematuria or the older patient).

\section{References}

1 Kitterer D, Guerzing K, Segerer S et al. Diagnostic impact of percutaneous renal biopsy. Clinical Nephrology 2015;84:311-22.

2 Prabhakar SS. Nephrology. In: Harward MP (ed). Medical secrets, 5th edn. Mosby, 2012:190-219.

3 National Institute for Health and Care Excellence. Urological cancers - recognition and referral. NICE, 2021. https://cks.nice.org.uk/ topics/urological-cancers-recognition-referral

4 National Institute for Health and Care Excellence. Chronic kidney disease in adults: assessment and management: Clinical guideline [CG182]. NICE, 2014. www.nice.org.uk/guidance/cg182

5 Cancer Research UK. Bladder cancer risk. Cancer Research UK, 2021. www.cancerresearchuk.org/health-professional/cancer-statistics/ statistics-by-cancer-type/bladder-cancer/risk-factors\#theading-Three

6 Cancer Research UK. Kidney cancer risk. Cancer Research UK, 2021. www.cancerresearchuk.org/health-professional/cancer-statistics/ statistics-by-cancer-type/kidney-cancer/risk-factors\#heading-Three

7 Anderson J, Fawcett D, Feehally ] et al. Joint consensus statement on the initial assessment of haematuria: Prepared on behalf of the renal association and British Association of Urological Surgeons. BAUS/RA, 2008:1-5.

8 McGregor D, Lynn K, Bailey R, Robson R, Gardner J. Clinical audit of the use of renal biopsy in the management of isolated microscopic hematuria. Clinical Nephrology 1998;49:345-8.

9 Richards N, Darby S, Howie A, Adu D, Michael J. Knowledge of renal histology alters patient management in over $40 \%$ of cases. Nephrology Dialysis Transplantation 1994;9:1255-9.

10 Topham P, Harper S, Furness P et al. Glomerular disease as a cause of isolated microscopic haematuria. QJM: An International Journal of Medicine 1994;87:329-35.

11 Vivante A, Afek A, Frenkel-Nir Y et al. Persistent asymptomatic isolated microscopic hematuria in Israeli adolescents and young adults and risk for end-stage renal disease. JAMA 2011;306:729-36.

12 Chan MM, Gale DP. Isolated microscopic haematuria of glomerular origin: clinical significance and diagnosis in the 21st century. Clin Med 2015:15:576.

13 Khanna R. Clinical presentation \& management of glomerular diseases: hematuria, nephritic \& nephrotic syndrome. Missouri Medicine 2011;108:33.

14 Floege J, Feehally J. Introduction to Glomerular Disease: Clinical Presentations. In: Floege J, Johnson RJ, Feehally J (eds). Comprehensive Clinical Nephrology, 4th edn. Mosby, 2010:193-207.

15 Kidney Disease: Improving Global Outcomes (KDIGO) CKD Work Group. KDIGO 2012 clinical practice guideline for the evaluation and management of chronic kidney disease. Kidney Int Suppl 2013;3:1-50. 
16 Fogazzi GB. Urinalysis. In: Feehally J, Floege J, Tonelli M, Johnson RJ (eds). Comprehensive Clinical Nephrology. Elsevier, 2018:26-39.

17 Ingold C], Bhatt H. Orthostatic proteinuria. StatPearls, 2021.

18 Korneti PG, Tasic VB, Jovanoska LK, Korneti BK. Difference between orthostatic and march functional proteinuria by application of stress tolerance test and SDS-PAGE. Clin Chim Acta 2000;297:145-53.

19 NHS Digital. National Diabetes Audit - Report 2 Complications and Mortality, 2017-18. NHS, 2019.

20 Hill C, Cardwell C, Patterson C et al. Chronic kidney disease and diabetes in the National Health Service: a cross-sectional survey of the UK National Diabetes Audit. Diabetic Medicine 2014;31:448-54.

21 Sharma SG, Bomback AS, Radhakrishnan ] et al. The modern spectrum of renal biopsy findings in patients with diabetes. Clinical Journal of the American Society of Nephrology 2013;8:1718-24.

22 Alicic RZ, Rooney MT, Tuttle KR. Diabetic kidney disease: challenges, progress, and possibilities. Clinical Journal of the American Society of Nephrology 2017:12:2032-45.

23 De Zeeuw D, Remuzzi G, Parving H-H et al. Proteinuria, a target for renoprotection in patients with type 2 diabetic nephropathy: lessons from RENAAL. Kidney International 2004;65:2309-20.

24 KDOQI. KDOQI clinical practice guidelines and clinical practice recommendations for diabetes and chronic kidney disease. Am J Kidney Dis 2007:49(2 Suppl 2):S12-154.

25 Liang S, Zhang X-G, Cai G-Y et al. Identifying parameters to distinguish non-diabetic renal diseases from diabetic nephropathy in patients with type 2 diabetes mellitus: a meta-analysis. PloS One 2013;8:e64184.

26 Patel M, Clarke AM, Bruce IN, Symmons DP. The prevalence and incidence of biopsy-proven lupus nephritis in the UK: Evidence of an ethnic gradient. Arthritis Rheum 2006;54:2963-9.

27 Cervera R, Khamashta MA, Font ] et al. Systemic lupus erythematosus: clinical and immunologic patterns of disease expression in a cohort of 1,000 patients. The European Working Party on Systemic Lupus Erythematosus. Medicine 1993;72:113-24.

28 Moroni G, Vercelloni PG, Quaglini S et al. Changing patterns in clinical-histological presentation and renal outcome over the last five decades in a cohort of 499 patients with lupus nephritis. Annals of the Rheumatic Diseases 2018;77:1318-25.

29 Fanouriakis A, Kostopoulou M, Cheema K et al. 2019 update of the joint European League Against Rheumatism and European Renal Association-European Dialysis and Transplant Association (EULAR/ ERA-EDTA) recommendations for the management of lupus nephritis. Annals of the Rheumatic Diseases 2020;79:713-23.

30 Kidney Disease: Improving Global Outcomes (KDIGO). KDIGO Clinical Practice Guideline for Glomerulonephritis. Kidney International 2012;2 (suppl 2):139-274.

31 Moroni G, Depetri F, Ponticelli C. Lupus nephritis: when and how often to biopsy and what does it mean? Journal of Autoimmunity 2016;74:27-40.

32 Illei G, Takada K, Parkin D et al. Renal flares are common in patients with severe proliferative lupus nephritis treated with pulse immunosuppressive therapy: long-term followup of a cohort of 145 patients participating in randomized controlled studies. Arthritis Rheum 2002:46:995-1002.

33 Narváez J, Ricse M, Gomà M et al. The value of repeat biopsy in lupus nephritis flares. Medicine 2017;96:e7099.

34 Moroni G, Pasquali S, Quaglini S et al. Clinical and prognostic value of serial renal biopsies in lupus nephritis. American Journal of Kidney Diseases 1999;34:530-9.

35 Kolhe N, Muirhead A, Wilkes S, Fluck R, Taal M. The epidemiology of hospitalised acute kidney injury not requiring dialysis in England from 1998 to 2013: retrospective analysis of hospital episode statistics. International Journal of Clinical Practice 2016;70:330-9.

36 NHS Digital. Health Survey for England 2016: Kidney and liver disease. NHS, 2017. https://digital.nhs.uk/data-and-information/ publications/statistical/health-survey-for-england/health-survey-forengland-2016

37 Glassock RJ, Rule AD. The implications of anatomical and functional changes of the aging kidney: with an emphasis on the glomeruli. Kidney International 2012;82:270-7.

38 Haas M, Spargo BH, Wit E-JC, Meehan SM. Etiologies and outcome of acute renal insufficiency in older adults: a renal biopsy study of 259 cases. American Journal of Kidney Diseases 2000;35:433-47.

39 Navaratnarajah A, Sambasivan K, Cook TH et al. Predicting longterm renal and patient survival by clinicopathological features in elderly patients undergoing a renal biopsy in a UK cohort. Clinical Kidney Journal 2019;12:512-20.

40 Bomback AS, Appel GB, Radhakrishnan ] et al. ANCA-associated glomerulonephritis in the very elderly. Kidney International 2011; 79:757-64.

41 Lees JS, McQuarrie EP, Mordi N et al. Risk factors for bleeding complications after nephrologist-performed native renal biopsy. Clinical Kidney Journal 2017:10:573-7.

42 Nair R, Bell JM, Walker PD. Renal biopsy in patients aged 80 years and older. American Journal of Kidney Diseases 2004;44:618-26.

43 Kyle R, Rajkumar SV. Criteria for diagnosis, staging, risk stratification and response assessment of multiple myeloma. Leukemia 2009;23:3-9.

44 Leung N, Rajkumar SV. Renal manifestations of plasma cell disorders. American Journal of Kidney Diseases 2007;50:155-65.

45 Rajkumar SV, Dimopoulos MA, Palumbo A et al. International Myeloma Working Group updated criteria for the diagnosis of multiple myeloma. Lancet Oncology 2014;15:e538-48.

46 Knudsen LM, Hippe E, Hjorth M, Holmberg E, Westin J. Renal function in newly diagnosed multiple myeloma-a demographic study of 1353 patients. European Journal of Haematology 1994; 53:207-12.

47 Dimopoulos MA, Kastritis E, Terpos E et al. International Myeloma Working Group recommendations for the diagnosis and management of myeloma-related renal impairment. Journal of Clinical Oncology 2016;34:1544-57.

48 Favà A, Fulladosa X, Montero N et al. Treatment of multiple myeloma with renal involvement: the nephrologist's view. Clinical Kidney Journal 2018;11:777-85.

49 Hogan J], Alexander MP, Leung N. Dysproteinemia and the kidney: core curriculum 2019. American Journal of Kidney Diseases 2019;74:822-36.

50 Fermand J-P, Bridoux F, Kyle RA et al. How I treat monoclonal gammopathy of renal significance (MGRS). Blood 2013;122:3583-90.

51 Ecotière L, Thierry A, Debiais-Delpech C et al. Prognostic value of kidney biopsy in myeloma cast nephropathy: a retrospective study of 70 patients. Nephrology Dialysis Transplantation 2016;31:6472.

52 Kumar S, Paiva B, Anderson KC et al. International Myeloma Working Group consensus criteria for response and minimal residual disease assessment in multiple myeloma. Lancet Oncology 2016;17: e328-46.

53 Palladini G, Dispenzieri A, Gertz MA et al. New criteria for response to treatment in immunoglobulin light chain amyloidosis based on free light chain measurement and cardiac biomarkers: impact on survival outcomes. Journal of Clinical Oncology 2012;30:4541-9.

54 Dattani R, Corbett RW, Galliford ] et al. The effect of kidney biopsy on glomerular filtration rate: a frequent patient concern. American Journal of Nephrology 2020;51:903-6.

55 Poggio ED, McClelland RL, Blank KN et al. Systematic review and meta-analysis of native kidney biopsy complications. Clinical Journal of the American Society of Nephrology 2020;15:1595602.

56 Shidham GB, Siddiqi N, Beres JA et al. Clinical risk factors associated with bleeding after native kidney biopsy. Nephrology 2005; 10:305-10. 
57 Lin W, Yang Y, Wen Y, Chang C. Outpatient versus inpatient renal biopsy: a retrospective study. Clin Nephrol 2006;66:17-24.

58 Pombas B, Rodríguez E, Sánchez ] et al. Risk factors associated with major complications after ultrasound-guided percutaneous renal biopsy of native kidneys. Kidney and Blood Pressure Research 2020;45:122-30.
Address for correspondence: Dr Matthew Graham-Brown, Department of Cardiovascular Sciences, University of Leicester, Leicester LE1 9HN, UK.

Email:mgb23@leicester.ac.uk

Twitter: @DrMattGB; @KatherineHullI; @SAdenwalla 